\title{
Outcomes of deliveries by family physicians or obstetricians: a population-based cohort study using an instrumental variable
}

\author{
Kris Aubrey-Bassler MSc MD, Richard M. Cullen BSc, Alvin Simms PhD, Shabnam Asghari MD PhD, \\ Joan Crane MSc MD, Peizhong Peter Wang MD PhD, Marshall Godwin MSc MD
}

CMAJ Podcasts: author interview at https://soundcloud.com/cmajpodcasts/141633-res

\begin{abstract}
Background: Previous research has suggested that obstetric outcomes are similar for deliveries by family physicians and obstetricians, but many of these studies were small, and none of them adjusted for unmeasured selection bias. We compared obstetric outcomes between these provider types using an econometric method designed to adjust for unobserved confounding.
\end{abstract}

Methods: We performed a retrospective population-based cohort study of all Canadian (except Quebec) hospital births with delivery by family physicians and obstetricians at more than 20 weeks gestational age, with birth weight greater than $500 \mathrm{~g}$, between Apr. 1, 2006, and Mar. 31, 2009. The primary outcomes were the relative risks of in-hospital perinatal death and a composite of maternal mortality and major morbidity assessed with multivariable logistic regression and instrumental variable-adjusted multivariable regression.
Results: After exclusions, there were 3600 perinatal deaths and 14394 cases of maternal morbidity among 799823 infants and 793053 mothers at 390 hospitals. For deliveries by family physicians $v$. obstetricians, the relative risk of perinatal mortality was 0.98 (95\% confidence interval $[\mathrm{Cl}]$ 0.85-1.14) and of maternal morbidity was $0.81(95 \% \mathrm{Cl} 0.70-0.94)$ according to logistic regression. The respective relative risks were $0.97(95 \% \mathrm{Cl} 0.58-1.64)$ and $1.13(95 \% \mathrm{Cl} 0.65-1.95)$ according to instrumental variable methods.

Interpretation: After adjusting for both observed and unobserved confounders, we found a similar risk of perinatal mortality and adverse maternal outcome for obstetric deliveries by family physicians and obstetricians. Whether there are differences between these groups for other outcomes remains to be seen.
Competing interests: None declared

This article has been peer reviewed.

Accepted: July 3, 2015

Online: Aug. 24, 2015

Correspondence to: Kris Aubrey-Bassler; kaubrey@mun.ca

CMAJ 2015. DOI:10.1503/ cmaj. 141633
$\mathrm{O}$ ver the past several decades in Canada, obstetric deliveries have increasingly been attended by specialist obstetricians rather than family physicians. ${ }^{1}$ Although specialized care is beneficial for high-risk mothers and their infants, ${ }^{2-4}$ there are concerns that it might increase risk for women whose deliveries could be safely managed without a specialized approach. Most prior studies have concluded that obstetric outcomes between family physicians and obstetricians are similar, but many of these studies were small, and none of them adjusted for unmeasured factors that might affect both the choice of delivery provider and outcomes. ${ }^{5-8}$

Obstetric risk is typically divided between providers, with family physicians and obstetricians sharing the lowest-risk patients, obstetricians caring for moderate-risk patients, and subspecialized perinatologists caring for the highest-risk individuals. Although traditional statistical methods can be used to adjust for observed differences between these groups, they cannot be used to adjust for unobserved differences. For example, the presence of gestational diabetes mellitus is usually noted, but its severity is often not coded in administrative databases. Women with mild diabetes mellitus are usually eligible for delivery by family physicians, but those with severe diabetes (and the attendant increased risk of adverse outcome) are not. There are many prominent examples where traditional analyses of observational data produced results that were subsequently refuted by randomized trials, presumably because of these unmeasured or unknown factors that also affect treatment decisions or outcomes. ${ }^{4,9-11}$

The instrumental variable method from the field of econometrics is a technique designed to control for unmeasured covariates in regression 
analyses. Results from instrumental variablecontrolled observational analyses of the effect of angiography after myocardial infarction ${ }^{9}$ and of long-acting bronchodilators on asthma control ${ }^{12}$ closely approximated those of randomized controlled trials, whereas analyses using traditional statistical methods differed substantially. Instrumental variable analyses of obstetric data have shown that traditional statistical approaches significantly underestimate the mortality benefit of high-volume hospitals for high-risk neonates. ${ }^{4}$ The objective of the current study was to compare perinatal mortality and maternal morbidity and mortality for deliveries by family physicians and obstetricians using instrumental variable methodology.

\section{Methods}

\section{Study design, data sources and population}

We collected maternal and neonatal data for all of Canada (except the province of Quebec) from the Canadian Institute for Health Information (CIHI) Discharge Abstracts Database for deliveries between Apr. 1, 2006, and Mar. 31, 2009. This database has been used for several surveillance reports $^{13-15}$ and numerous studies ${ }^{16-22}$ of obstetric outcomes, and it captures clinically significant diagnoses with high sensitivity and specificity. ${ }^{23,24}$

We linked these records to Statistics Canada census-derived socioeconomic information using the maternal residential postal code. ${ }^{25} \mathrm{We}$ also accessed records for discharged and readmitted infants. Infants with birth weight less than $500 \mathrm{~g}$ or gestational age less than 20 weeks at delivery were excluded, for consistency with other studies. ${ }^{2,26} \mathrm{We}$ analyzed maternal data independently, whereas neonatal records were linked to the corresponding maternal record using a linkage variable provided by CIHI or by probabilistic linkage using other variables. We excluded infant records that could not be matched to a single mother. This research was approved by the provincial Health Research Ethics Authority (Newfoundland and Labrador).

\section{Outcomes}

Perinatal mortality and a composite of maternal morbidity and mortality were the primary outcome measures. The perinatal outcome was defined as in-hospital death after 20 weeks' gestational age, and the maternal outcome was defined similarly to Joseph and associates ${ }^{17}$ with some additions from other papers. ${ }^{21,27}$ Although most definitions of perinatal mortality exclude death after 7 days of age, we included such deaths for infants who were continuously hospitalized (including transfers), to protect against bias associated with technologically advanced hospitals and providers who had the capability to keep infants alive longer than that. The codes used to define the outcomes are listed in Appendices 1 and 2 (available at www.cmaj.ca/lookup/ suppl/doi:10.1503/cmaj.141633/-/DC1).

\section{Group assignment}

The study database included fields recording the types of providers involved in delivering care during the admission and the role that each played. Mothers were enrolled in the family physician group if that provider was listed at any point as the most responsible provider. This approach appropriately assigns patients for whom a family physician delivery was planned but who experienced intrapartum complications requiring transfer to an obstetrician or other provider (e.g., for cesarean section). We believe that this is a conservative assignment of patients that may bias against family physicians in some models where care is shared between these providers and obstetricians. In these models, high-risk patients for whom an obstetrician delivery is planned are often admitted under the family physician.

Mothers attended by midwives were identified in a similar fashion but were excluded from analyses. The remaining patients were categorized into the obstetrician group if the delivery provider was an obstetrician, and any records remaining after these assignments were excluded. We conducted sensitivity analyses assigning patients solely to the practitioner coded as most responsible or to the practitioner coded as the delivery provider. We conducted an additional sensitivity analysis including midwife deliveries in the sample.

\section{Instrumental variable}

An instrumental variable is one that predicts the receipt of treatment but is not directly associated with outcomes, except through its effect on treatment. We used the proportion of women living within the catchment of the woman's local hospital who were delivered by a family physician as an instrumental variable. We compared comorbidities across quintiles of the instrumental variable and calculated $F$ statistics and partial correlations as measures of its suitability. We also confirmed that the $F$ statistics exceeded the values defining an acceptable instrument. ${ }^{28}$ Additional information is provided in Appendix 3 (available at www.cmaj.ca/ lookup/suppl/doi:10.1503/cmaj.141633/-/DC1).

\section{Hospital and other covariates}

We assigned women to hospital catchment areas using the Hospital Referral Region method, ${ }^{29}$ except that we did not adjust catchment areas for 
geographic contiguity. Briefly, this method assigns a postal code to a given hospital when a plurality of patients living within that postal code is admitted to that hospital for their acute inpatient care. All (not just obstetric) visits to acute care hospitals for the study period were used to assign postal codes in this fashion.

Each hospital in our data set was assigned a service level according to Canadian Pediatric Society guidelines. ${ }^{30}$ For additional details, see Appendix 3. We conducted sensitivity analyses including only a tertiary hospital term and excluding delivery hospital level entirely from the regression analyses. We also conducted sensitivity analyses including cesarean section or procedural delivery (forceps or vacuum delivery) as a covariate in our regression models.

\section{Statistical analysis}

Analyses were conducted using Stata software, version 13.1 (StataCorp LLP). We measured the effect of delivery provider on our outcomes initially with bivariable logistic regression, then with multivariable logistic regression controlling for the covariates listed in Appendix 3. We did not adjust logistic models for the instrumental variable. We estimated risk ratios from these logistic models as described previously. ${ }^{31} \mathrm{We}$ also used 3 different instrumental variable meth- ods because results sometimes vary with different approaches. ${ }^{32}$ For the first method, we divided the data into quintiles of the instrumental variable and estimated the relative risk of the outcome for each quintile, as described previously. ${ }^{31}$ We then analyzed the data using 2-stage least-squares regression, including a method that is robust to weak instruments, ${ }^{33}$ and the generalized method of moments (Stata syntax, including analytic first derivatives, as published previously $^{34}$ ). All analyses were adjusted for clustering at the delivery hospital. For an expanded description of the instrumental variable analyses, please see Appendix 3.

\section{Results}

The cohorts and their exclusions are described in Figure 1. Of the 859180 neonatal records accessed (before exclusions), $69.4 \%$ had delivery by obstetricians, $26.2 \%$ by family physicians and $3.7 \%$ by midwives. Across quintiles, midwives delivered $3.7 \%, 4.5 \%, 4.1 \%, 3.4 \%$ and $2.8 \%$ of the infants, respectively; the breakdown by quintile of deliveries by family physicians and obstetricians is provided in Table 1. For the remaining $0.7 \%$ of infants, either delivery was by another provider type or information on provider type was missing. We identified a total of 3600

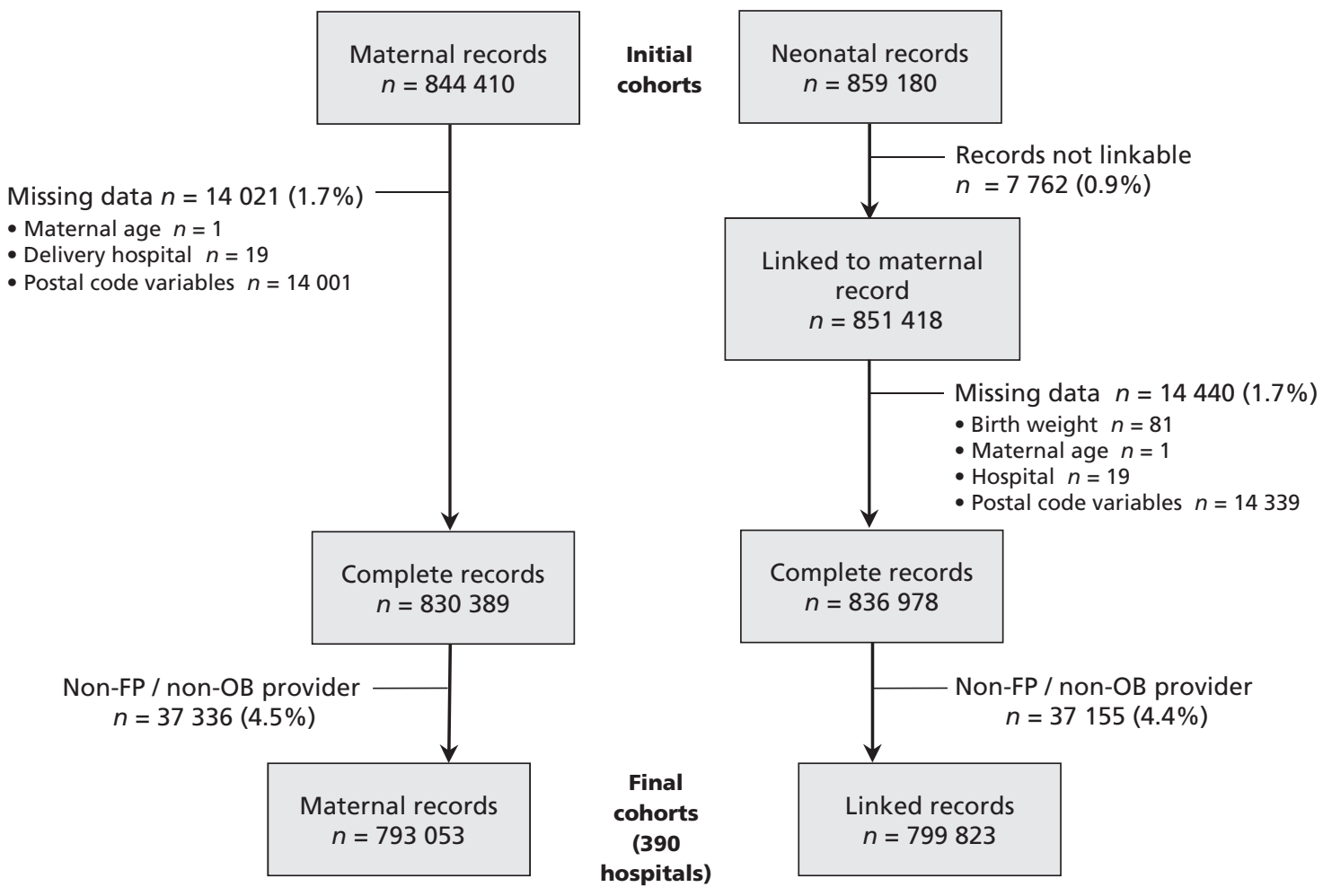

Figure 1: Flow diagram for study cohort. FP = family physician, $O B=$ obstetrician. 
(0.45\%) perinatal deaths and 14394 (1.82\%) cases of maternal morbidity and mortality during the study period. Selected characteristics of the study population, delivery providers and hospitals are listed in Table 1. Notably, there was an apparent positive correlation between predicted risk of maternal morbidity and mortality and the proportion of the population who identified as $\mathrm{Ab}$ original, which was a significant predictor of perinatal mortality (data not shown).

\section{Strength of instrumental variable}

Our instrumental variable predicted a wide range in the mean percentage of deliveries by a family physician (4.2\% to $67.7 \%$ across quintiles). Whereas there were some differences in measured covariates across these quintiles (Table 1), there was essentially no correlation between mean perinatal mortality $\left(r^{2}=7.3 \times 10^{-6}\right)$ or maternal morbidity and mortality $\left(r^{2}=0.011\right)$ and the instrumental variable, a required characteristic to ensure

\begin{tabular}{|c|c|c|c|c|c|c|}
\hline \multirow[b]{2}{*}{ Characteristic } & \multicolumn{6}{|c|}{ Quintile of regional FP delivery rate; \% of cases* } \\
\hline & 1 & 2 & 3 & 4 & 5 & All \\
\hline \multicolumn{7}{|l|}{ Delivery provider } \\
\hline FP (instrumental variable) & 4.2 & 9.0 & 16.3 & 38.9 & 67.7 & 27.3 \\
\hline Obstetrician & 95.8 & 91.1 & 83.7 & 61.1 & 32.3 & 72.7 \\
\hline Annual volume, mean & 265 & 274 & 244 & 179 & 98 & 212 \\
\hline \multicolumn{7}{|l|}{ Delivery hospital } \\
\hline Level 3 (with tertiary NICU) & 9.4 & 26.3 & 35.5 & 37.8 & 23.7 & 26.5 \\
\hline Annual volume, mean & 2633 & 3729 & 2836 & 3492 & 2043 & 2944 \\
\hline \multicolumn{7}{|l|}{ Maternal } \\
\hline No. of records & 153108 & 159812 & 162388 & 155939 & 161806 & 793053 \\
\hline Age, yr, mean & 29.3 & 30.5 & 29.0 & 29.3 & 28.3 & 29.3 \\
\hline Income, $\$$, meant & 27478 & 28881 & 28073 & 26677 & 26651 & 27560 \\
\hline Education, some high schoolt & 83.0 & 86.4 & 83.6 & 83.6 & 80.2 & 83.4 \\
\hline Ethnicity, Aboriginal† & 3.5 & 3.5 & 4.8 & 5.9 & 10.8 & 5.7 \\
\hline Urban (CMA or CA)† & 86.6 & 82.6 & 85.5 & 78.5 & 60.0 & 80.8 \\
\hline Cesarean section & 29.6 & 29.0 & 28.7 & 29.0 & 28.9 & 29.0 \\
\hline Prior cesarean section & 13.2 & 12.9 & 12.6 & 12.9 & 13.0 & 12.9 \\
\hline Diabetes mellitus type 1 & 0.28 & 0.26 & 0.29 & 0.25 & 0.24 & 0.26 \\
\hline Diabetes mellitus type 2 & 0.27 & 0.30 & 0.38 & 0.27 & 0.31 & 0.31 \\
\hline Gestational diabetes mellitus & 4.5 & 4.8 & 4.4 & 5.9 & 4.0 & 4.7 \\
\hline Eclampsia & 0.09 & 0.06 & 0.07 & 0.04 & 0.06 & 0.06 \\
\hline $\mathrm{PIH}$ & 5.7 & 5.5 & 6.5 & 6.3 & 6.5 & 6.1 \\
\hline HIV & 0.05 & 0.05 & 0.07 & 0.08 & 0.04 & 0.06 \\
\hline $\begin{array}{l}\text { Predicted maternal morbidity } \\
\text { and mortality, per } 1000 \ddagger\end{array}$ & 16.5 & 18.1 & 17.4 & 17.1 & 21.5 & 18.2 \\
\hline \multicolumn{7}{|l|}{ Neonatal } \\
\hline No. of records & 154485 & 161694 & 165297 & 155606 & 162741 & 799823 \\
\hline Sex, male & 51.2 & 51.2 & 51.3 & 51.4 & 51.2 & 51.3 \\
\hline GA, wk, mean & 38.7 & 38.8 & 38.7 & 38.8 & 38.9 & 38.8 \\
\hline Weight, g, mean & 3357 & 3340 & 3376 & 3363 & 3416 & 3371 \\
\hline Twin & 2.6 & 3.1 & 2.9 & 3.0 & 2.6 & 2.9 \\
\hline Triplet or greater & 0.10 & 0.13 & 0.09 & 0.06 & 0.06 & 0.09 \\
\hline Congenital anomaly & 3.0 & 2.9 & 3.3 & 3.3 & 2.8 & 3.1 \\
\hline Abruptio placenta & 0.12 & 0.10 & 0.31 & 0.17 & 0.14 & 0.17 \\
\hline PROM & 0.30 & 0.26 & 1.39 & 0.64 & 0.32 & 0.59 \\
\hline $\begin{array}{l}\text { Predicted perinatal mortality, } \\
\text { per } 1000 \neq\end{array}$ & 4.8 & 4.5 & 4.5 & 4.4 & 4.3 & 4.5 \\
\hline 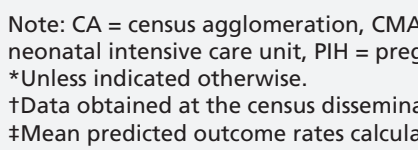 & $\begin{array}{l}\text { nsus metrop } \\
\text {-y-induced } h \\
\text { level. } \\
\text { rom a logist }\end{array}$ & $\begin{array}{l}\text { an area, } \mathrm{FH} \\
\text { rtension, } \mathrm{P} \\
\text { egression } \mathrm{n}\end{array}$ & $\begin{array}{l}\text { amily phys } \\
M=\text { prema }\end{array}$ & $\begin{array}{l}\text { an, } \mathrm{GA}=\mathrm{ge} \\
\text { e rupture o } \\
\text { II covariate }\end{array}$ & $\begin{array}{l}\text { tional age, } 1 \\
\text { embranes. } \\
\text { cept deliver }\end{array}$ & $\begin{array}{l}U= \\
\text { rovider. }\end{array}$ \\
\hline
\end{tabular}


unbiased results. The Kleibergen-Paap $F$ statistics for our instrumental variable far exceeded the value necessary to define a strong instrument ${ }^{28}$ (see Appendix 3). The partial correlation coefficient between the delivery provider and the instrumental variable was 0.55 for both the neonatal and maternal analyses, indicating that $30 \%$ of the variation in the rate of delivery by family physicians was explained by the instrumental variable, which is also a marker of a strong instrument.

\section{Effect of family physician as delivery provider}

The risk ratios for the outcomes across the different family physician quintiles are presented in Table 2. Ad hoc risk ratios from the traditional 2-stage least-squares models were 0.95 for perinatal mortality and 1.01 for the maternal outcome, and the results were identical using methods that were robust to weak instruments. ${ }^{33}$ Results from logistic and generalized method of moments analyses are presented in Table 3. The sensitivity analyses described in the Methods section changed neither the direction of the estimated effects (risk ratio greater than or less than 1.0) nor the statistical significance of the association (data not shown).

\section{Interpretation}

Using a statistical method that controls for both observed and unmeasured or unknown factors affecting obstetric outcomes, we found no difference in the risk of perinatal mortality or maternal morbidity and mortality between deliveries by family physicians and those by obstetricians. However, because of the limitations of this statistical method, the confidence intervals around the risk ratios are wide. These findings build on previous work that also supports the safety of obstetric delivery by family physicians. $^{5-8}$ The current study is among the largest on this topic to date and strengthens these earlier findings by including an adjustment for unmeasured selection bias.

It is common to assume that more specialized or higher-volume medical care will result in improved outcomes. This has been most convincingly shown for highly technical, relatively uncommon procedures such as pancreatectomy, esophagectomy and elective repair of abdominal aortic aneurysm. ${ }^{35}$ Similarly, the obstetric literature has consistently shown that outcomes for high-risk newborns and mothers are best at higher-volume, more specialized hospitals $;^{2-4}$

Table 2: Risk ratios for outcomes across FP delivery quintiles

\begin{tabular}{|lccccc|}
\hline \multicolumn{5}{c}{ Quintile of regional FP delivery rate; ARR (95\% CI) } \\
\cline { 2 - 6 } Outcome & 1 & 2 & 3 & 4 & 5 \\
\hline $\begin{array}{l}\text { Perinatal } \\
\text { mortality }\end{array}$ & 1.00 & $1.05(0.92-1.21)$ & $0.84(0.71-0.98)$ & $0.97(0.79-1.18)$ & $0.90(0.75-1.08)$ \\
\hline $\begin{array}{l}\text { Maternal } \\
\text { morbidity and } \\
\text { mortality }\end{array}$ & 1.00 & $1.09(0.85-1.41)$ & $0.99(0.78-1.26)$ & $0.96(0.74-1.24)$ & $1.08(0.82-1.43)$ \\
\hline $\begin{array}{l}\text { Note: ARR = adjusted risk ratio, Cl = confidence interval, FP = family physician. } \\
\text { *Adjusted for all comorbidities listed in Appendix 1 (available at www.cmaj.ca/lookup/suppl/doi:10.1503/cmaj.141633/-/DC1). }\end{array}$ \\
\hline
\end{tabular}

Table 3: Effect of delivery by family physicians on perinatal mortality and maternal morbidity and mortality using logistic and IV-adjusted regression

\begin{tabular}{|lcc|}
\hline & \multicolumn{2}{c|}{ Outcome; RR $(95 \% \mathrm{Cl})$} \\
\cline { 2 - 3 } Method & Perinatal mortality & Maternal morbidity and mortality \\
\hline Logistic regression & $0.43(0.34-0.55)$ & $0.81(0.68-0.96)$ \\
\hline Unadjusted & $0.98(0.85-1.14)$ & $0.81(0.70-0.94)$ \\
\hline Multivariable adjusted* & $0.80(0.40-1.57)$ & $1.49(0.77-2.87)$ \\
\hline GMM (IV adjusted) & $0.97(0.58-1.64)$ & $1.13(0.65-1.95)$ \\
\hline $\begin{array}{l}\text { Unadjusted } \\
\text { Multivariable adjusted* }\end{array}$ & & \\
\hline $\begin{array}{l}\text { Note: CI = confidence interval, GMM = generalized method of moments, IV = instrumental variable, RR = risk ratio. } \\
\text { *Multivariable adjusted models were controlled for all comorbidities listed in Appendix 1 (available at www.cmaj.ca/lookup/ } \\
\text { suppl/doi:10.1503/cmaj.141633/-IDC1). }\end{array}$ \\
\hline
\end{tabular}


however, findings from the literature on low-risk deliveries are variable..$^{26,36-39}$

Although there are exceptions, several studies have found differences between family physicians and obstetricians in the use of invasive procedures such as forceps, vacuum, episiotomy and cesarean section for the management of labour, ${ }^{6-8}$ and research suggests that this more invasive care may be harmful to mothers and infants..$^{40,41} \mathrm{We}$ chose not to adjust for procedures in our primary analyses because we wanted to capture differences in this covariate within the specialty variable. It is tempting to assume that the improved outcomes expected because of specialty training were offset in our study by an increased risk associated with a higher procedure rate. However, we observed a nonlinear trend in rates of cesarean section across the family physician quintiles (Table 1). We also conducted sensitivity analyses including cesarean section or procedural delivery as a covariate in our regression models, and these adjustments did not change our primary findings. We will further explore the effect of procedure use on obstetric outcomes in a forthcoming paper.

Instrumental variables analyses measure outcomes for the "marginal" population, which in our study consisted of those patients who would be delivered by a family physician in some jurisdictions but not in others. Although some clearly high-risk patients may on occasion be delivered by a family physician if labour proceeds precipitously and no obstetricians are available, most of these patients would have been delivered by a specialist, and our estimates of treatment effect do not apply to them. Because of this, instrumental variableadjusted analyses do not easily translate into decisions that can be applied to an individual expectant mother, except if she fits the profile of the marginal population where she intends to deliver. Instead, instrumental variable-adjusted findings are more relevant for policy-makers who are making decisions at the health system level that will affect the choice of one treatment over another.

Some authors have found large differences between risk estimates with these 2 types of analyses; $;, 12$ however, in our analyses, the results for instrumental variable-adjusted and comprehensively adjusted traditional models differed relatively little, particularly for the perinatal mortality outcome. This suggests that the covariates included in our analyses captured most of the variance associated with differences between our groups and/or that the indications for delivery by an obstetrician are relatively clear. Traditional statistical methods are more likely to produce unbiased estimates under one or both of these conditions.

\section{Limitations}

Several limitations of our study merit discussion. We excluded midwife deliveries from our analyses to obtain a "cleaner" comparison. Although midwives attend a substantial proportion of lowrisk deliveries in some regions of the country, our statistical method adjusts for the between-group risk difference exacerbated by this exclusion. Including midwife deliveries in the sample in a sensitivity analysis did not alter our final conclusion. We have not presented separate analyses for the midwife group because they represent a small proportion of our sample overall, and there is much less variability in the proportion of deliveries by midwives across regions. Thus, the potential for bias, even with instrumental variableadjusted analyses, is greater with this group. Furthermore, we did not have access to data for home deliveries, which make up a very small proportion of deliveries overall but often a substantial proportion of deliveries by midwives, and patients who choose a home delivery likely differ systematically from those who choose hospital delivery. Because home deliveries accounted for less than $1.5 \%$ of deliveries in Canada during our study period, ${ }^{42}$ we do not feel that this exclusion significantly biases the analyses presented here.

We did not attempt to measure perinatal outcomes other than death. Infants may benefit in other ways from delivery by obstetricians or family physicians, particularly the lower-risk infant population in whom the risk of death is very low. It is difficult to imagine important maternal complications that are not captured in our comprehensive definition of maternal morbidity. However, there is a small likelihood that we did not observe differences between groups in diagnoses that arose after hospital discharge, because we did not attempt to access maternal readmission records (as we did for the neonates).

Although our chosen variable met the commonly used thresholds for a strong instrument, there was some variability in measured covariates across quintiles of this variable (Table 1), which raises the possibility that unobserved covariates also vary across these levels. In particular, although the predicted risk of perinatal mortality was relatively consistent across quintiles, there was an apparent positive correlation between predicted risk of maternal morbidity and mortality and the instrument (although the $r^{2}$ was very low). There was also a similar trend across quintiles in the proportion of the population who identified as Aboriginal, which was a significant predictor of perinatal mortality. Although we adjusted for these covariates, insofar as unobserved covariates vary in a similar pattern, the trend we observed would tend to bias results against family physicians. Addi- 
tional unobserved covariates with a similar distribution may explain the trend toward worse maternal outcomes that we observed in the instrumental variable-adjusted analyses, despite the lower risk observed in logistic analyses (Table 3).

Finally we acknowledge that these data are now somewhat out of date. It is possible that there has been a cultural shift resulting in a change in the proportion of deliveries by family physicians or midwives in recent years. In addition, the increasing attention paid to procedural intervention in the obstetric literature (e.g., studies by Souza and colleagues ${ }^{40}$ and Villar and associates ${ }^{41}$ ) may have changed the management of deliveries by obstetricians.

\section{Conclusion}

In a large, population-based cohort of Canadian patients, we observed similar risks of perinatal mortality and adverse maternal outcome between obstetric deliveries by family physicians and those by obstetricians, using an econometric method designed to control for unmeasured bias. It remains to be seen whether there are differences between these groups in terms of other outcomes. Because of the analytical approach used, these findings apply only to mothers and infants who would be eligible for delivery by either family physicians or specialists in at least some jurisdictions of the country. These results do not apply to mothers who are consistently referred for delivery by obstetricians in all jurisdictions. Future research should explore the effect of different delivery providers on other outcomes and on health resource utilization.

\section{References}

1. Giving birth in Canada: providers of maternity and infant care. Ottawa: Canadian Institute for Health Information; 2004.

2. Phibbs CS, Baker LC, Caughey AB, et al. Level and volume of neonatal intensive care and mortality in very-low-birth-weight infants. N Engl J Med 2007;356:2165-75.

3. Cifuentes J, Bronstein J, Phibbs CS, et al. Mortality in low birth weight infants according to level of neonatal care at hospital of birth. Pediatrics 2002;109:745-51.

4. Wehby GL, Ullrich F, Xie Y. Very low birth weight hospital volume and mortality - an instrumental variables approach. Med Care 2012;50:714-21

5. Abenhaim HA, Welt M, Sabbah R, et al. Obstetrician or family physician: Are vaginal deliveries managed differently? J Obstet Gynaecol Can 2007;29:801-5.

6. Deutchman ME, Sills D, Connor PD. Perinatal outcomes: a comparison between family physicians and obstetricians. $J$ Am Board Fam Pract 1995;8:440-7.

7. Rosenblatt RA, Dobie SA, Hart LG, et al. Interspecialty differences in the obstetric care of low-risk women. Am J Public Health 1997;87:344-51.

8. Coco AS, Gates TJ, Gallagher ME, et al. Association of attending physician specialty with the cesarean delivery rate in the same patient population. Fam Med 2000;32:639-44

9. Stukel TA, Fisher ES, Wennberg DE, et al. Analysis of observational studies in the presence of treatment selection bias: effects of invasive cardiac management on AMI survival using propensity score and instrumental variable methods. JAMA 2007;297:278-85.

10. Anderson GL, Limacher M, Assaf AR, et al.; Women's Health Initiative Steering Committee. Effects of conjugated equine estrogen in postmenopausal women with hysterectomy: the Women's Health Initiative randomized controlled trial. JAMA 2004;291:1701-12.
11. Heart Protection Study Collaborative Group. MRC/BHF heart protection study of antioxidant vitamin supplementation in 20536 high-risk individuals: a randomised placebo-controlled trial. Lancet 2002;360:23-33.

12. Fang G, Brooks JM, Chrischilles EA. Comparison of instrumental variable analysis using a new instrument with risk adjustment methods to reduce confounding by indication. Am J Epidemiol 2012;175:1142-51.

13. Canadian perinatal health report -2008 edition. Ottawa: Public Health Agency of Canada; 2008.

14. Special report on maternal mortality and severe morbidity in Canada. Enhanced surveillance: the path to prevention. Ottawa: Health Canada; 2004.

15. Canadian perinatal health report 2003. Ottawa: Health Canada; 2003.

16. Wen SW, Huang L, Liston R, et al. Severe maternal morbidity in Canada, 1991-2001. CMAJ 2005;173:759-64.

17. Joseph KS, Liu S, Rouleau J, et al.; Maternal Health Study Group of the Canadian Perinatal Surveillance System. Severe maternal morbidity in Canada, 2003 to 2007: surveillance using routine hospitalization data and ICD-10CA codes. J Obstet Gynaecol Can 2010;32:837-46.

18. Joseph KS, Rouleau J, Kramer MS, et al.; Maternal Health Study Group of the Canadian Perinatal Surveillance System. Investigation of an increase in postpartum haemorrhage in Canada. BJOG 2007; 114:751-9.

19. Kramer MS, Rouleau J, Baskett TF, et al. Amniotic-fluid embolism and medical induction of labour: a retrospective, population-based cohort study. Lancet 2006;368:1444-8.

20. Liu S, Liston RM, Joseph KS, et al. Maternal mortality and severe morbidity associated with low-risk planned cesarean delivery versus planned vaginal delivery at term. CMAJ 2007; 176:455-60.

21. Wen SW, Rusen ID, Walker M, et al. Comparison of maternal mortality and morbidity between trial of labor and elective cesarean section among women with previous cesarean delivery. Am J Obstet Gynecol 2004;191:1263-9.

22. Aubrey-Bassler K, Newbery S, Kelly L, et al. Maternal outcomes of cesarean sections: Do generalists' patients have different outcomes than specialists' patients? Can Fam Physician 2007:53:2132-8.

23. Joseph KS, Fahey J; Canadian Perinatal Surveillance System. Validation of perinatal data in the discharge abstract database of the Canadian Institute for Health Information. Chronic Dis Can 2009; 29:96-100.

24. CIHI data quality study of the 2007-2008 Discharge Abstract Database. Ottawa: Canadian Institute for Health Information; 2010.

25. Wilkins R, Khan S. PCCF+ version $5 \mathrm{H}$ user's guide. 5th ed. Ottawa: Statistics Canada, Health Information and Research Division; 2011.

26. Goodman DC, Fisher ES, Little GA, et al. The relation between the availability of neonatal intensive care and neonatal mortality. N Engl J Med 2002;346:1538-44.

27. Roberts CL, Cameron CA, Bell JC, et al. Measuring maternal morbidity in routinely collected health data: development and validation of a maternal morbidity outcome indicator. Med Care 2008; 46:786-94.

28. Baum CF, Schaffer ME, Stillman S. Ivreg2: Stata module for extended instrumental variables/ 2SLS and GMM estimation. St. Louis: Federal Reserve Bank of St. Louis, Research Division, IDEAS; 2010. Available: http://ideas.repec.org/c/boc/bocode/ s425401.html (accessed 2015 May 28).

29. Dartmouth atlas of health care. Lebanon (NH): Dartmouth Institute for Health Policy and Clinical Practice; 2012. Available: www.dartmouthatlas.org/ (accessed 2012 Feb. 24).

30. Lee SK; CPS Fetus and Newborn Committee. Levels of neonatal care. Paediatr Child Health 2006;11:303-6.

31. Norton EC, Miller MM, Kleinman LC. Computing adjusted risk ratios and risk differences in Stata. Stata J 2013;13:492-509.

32. Stock JH, Wright JH, Yogo M. A survey of weak instruments and weak identification in generalized method of moments. $J$ Bus Econ Stat 2002;20:518-29.

33. Mikusheva A, Poi BP. Tests and confidence sets with correct size when instruments are potentially weak. Stata J 2006;6:335-47.

34. Palmer T, Harbord R, Clarke P, et al. Generalised method of moments estimations of structural mean models [presentation handout]. Meeting of UK Stata Users Group; 2011 Sep 15-16; London. Available: www.stata.com/meeting/uk11/abstracts/ UK11_palmer_handouts.pdf (accessed 2014 Oct 11).

35. Halm E, Lee C, Cassin M. Is volume related to outcome in health care? A systematic review and methodologic critique of the literature. Ann Intern Med 2002;137:511-20.

36. Moster D, Lie RT, Markestad T. Neonatal mortality rates in communities with small maternity units compared with those having larger maternity units. BJOG 2001;108:904-9. 
37. Heaphy PE, Bernard S. Maternal complications of normal deliveries: variation among rural hospitals. J Rural Health 2000; 16:139-147.

38. Phibbs CS, Bronstein JM, Buxton E, et al. The effects of patient volume and level of care at the hospital of birth on neonatal mortality. JAMA 1996;276:1054-9.

39. Rosenblatt RA, Reinken J, Shoemack P. Is obstetrics safe in small hospitals? Evidence from New Zealand's regionalised perinatal system. Lancet 1985;2:429-32.

40. Souza JP, Gulmezoglu AM, Lumbiganon P, et al. Caesarean section without medical indications is associated with an increased risk of adverse short-term maternal outcomes: the 2004-2008 WHO global survey on maternal and perinatal health. BMC Med 2010;8:71.

41. Villar J, Carroli G, Zavaleta N, et al. Maternal and neonatal individual risks and benefits associated with caesarean delivery: multicentre prospective study. BMJ 2007;335:1025-35.

42. CANSIM Table 102-4516: Live births and fetal deaths (stillbirths), by place of birth (hospital and non-hospital), Canada, provinces and territories [annual, for 2007-2011]. Ottawa: Statistics Canada; 2013. Available: www5.statcan.gc.ca/cansim/a26?lang=eng \&id=1024516 (accessed 2015 Aug. 5).

Affiliations: Primary Healthcare Research Unit, Discipline of Family Medicine (Aubrey-Bassler, Cullen, Asghari, Godwin), Department of Geography (Simms), Discipline of Obstetrics and Gynecology (Crane) and Division of Community Health and Humanities (Wang), Memorial University of Newfoundland, St. John's, NL.

Contributors: Kris Aubrey-Bassler was the principal investigator and contributed to initial conception, funding application, study design, data release protocol, data retrieval, statistical analysis, manuscript writing and editing. Richard Cullen contributed to data linkage and cleaning, correspondence with the data holder, ethics approval, manuscript preparation and revision. Alvin Simms contributed to data linkage and cleaning and to manuscript revision, and contributed geographic expertise. Shabnam Asghari contributed epidemiologic and administrative data expertise and participated in statistical analysis and manuscript revision. Joan Crane contributed obstetric expertise and participated in initial conception, funding application, study design and manuscript revision. Peter Wang contributed statistical expertise and participated in study design and manuscript revision. Marshall Godwin contributed family medicine expertise and participated in the funding application and manuscript revision. All authors approved the final version for publication and agree to act as guarantors of the work.

Funding: Funding for this study was provided to the research team through the Dr. A.R. Cox Research Grant via the Medical Research Endowment Fund at Memorial University of Newfoundland. The research reported was conducted independently of the funder.

Disclaimer: Parts of this article are based on data and information provided by the Canadian Institute for Health Information (CIHI). However, the analyses, conclusions, opinions and statements expressed herein are those of the authors and not those of CIHI.

Data sharing: The Canadian Institute for Health Information (CIHI) does not allow data sharing. However, upon appropriate approvals, raw data are available from CIHI, and we are able to share statistical software syntax files.

Acknowledgement: The authors thank Dr. James Rourke for comments on an earlier version of this paper. 